\title{
Protección gastrointestinal y antieméticos en radioterapia. II. Antieméticos
}

\section{Introducción}

Las náuseas y vómitos radioinducidos representan un problema importante para muchos pacientes oncológicos y pueden alterar su calidad de vida. Aunque la intensidad es menor que en los provocados por la quimioterapia, el fraccionamiento de la irradiación hace que los radioinducidos duren mas tiempo'. Mas aún, el uso de la radioquimioterapia concomitante está aumentando notablemente llegando a ser el tratamiento estandar para la mayoría de tumores sólidos (cabeza y cuello, gástrico, páncreas, cérvix, vejiga, etc) traduciéndose en una mayor incidencia de toxicidad aguda incluyendo las nausea y vómitos. La severidad de esos síntomas pueden impedir la administración completa del esquema terapéutico y por tanto reducir las posibilidades de cura.

Las náuseas y vómitos radioinducidos típicamente ocurren de forma aguda (en las primeras 24 horas tras la administración de la radioterapia). Si bien algunos pacientes presentan emesis retardada, esta es mas típica de la inducida por quimioterápicos. La incidencia y severidad ha sido mejorada por las mejoras en la planificación de la irradiación, dando como resultados una disminución del volumen sano a incluir en el tratamiento especialmente torácico, abdomino y pélvico. A pesar de ello un tratamiento estandar en estas localizaciones implica 30-35 fracciones en 6-8 semanas y ello conlleva la aparición de las náuseas y vómitos ${ }^{2}$.

La emetogenicidad de la radioterapia está relacionada con el volumen irradiado2, 3 . El 80-90\% de pacientes sometidos a una irradiación corporal total (TBI) y el $50-80 \%$ sometidos a irradiación abdominal superior sufren emesis, al igual que en otras localizaciones ${ }^{4,5}$. El impacto sobre la calidad de vida (QOL) en estos pacientes es reflejado en un estudio donde tras 10 o mas fracciones de irradiación sobre el abdomen, la presencia de nauseas, vómitos, diarrea y anorexia siguen presentes tras 5 semanas de finalizada la irra-

Servicio de Oncología Radioterápica

Hospital Universitario San Joan

Alicante. España diación ${ }^{6}$. Además pueden aparecer otros efectos, tales como mucositis, pérdida de apetito y cansancio que ayudan al deterioro general del paciente ${ }^{7}$. Además, las nauseas y vomitos radioinducidos alteran la calidad de vida por otras vías, al poder producir deshidratación, alteraciones hidroelectrolíticas y malnutrición ${ }^{8}$. También son importantes las consideraciones del tratamiento. Un estudio italiano ${ }^{5}$ muestra que el $56 \%$ de 914 pacientes sometidos a radioterapia presentaron emesis los mayores de 60 años. Resultados similares han sido publicados en otro estudio9. Basado en ello debemos tener en cuenta que los pacientes mayores de 60 años son mas susceptibles a presentar vómitos radioinducidos.

\section{Terapia antiemética profiláctica en las náuseas y vómitos radioinducidos}

Existe una clara evidencia ${ }^{3,10}$ de que el uso de antagonistas de la 5-HT3 es la terapia recomendada para las náuseas y vómitos inducidos por la quimioterapia. En cambio, la literatura médica es mas pobre en cuanto a estudios randomizados para demostrar la efectividad en las náusea y vómitos radioinducidos, y los pocos estudios publicados se centran en la TBI. Sin embargo se recomienda el uso profiláctico de antagonistas de la 5-HT3 asociado o no a esteroides en pacientes sometidos a regímenes de radioterapia con moderado ó alto poder emetógeno ${ }^{3,10}$.

Los antagonistas granisetrón y ondansetrón están permitidos en muchos países para el tratamiento de las nauseas y vómitos radioinducidos. Otros, dolasetrón y tropisetrón están autorizados para las náuseas y vómitos quimioinducidos y no para los radioinducidos.

Dado que actualmente es anecdótico el uso de radioterapia exclusiva, el uso de quimioterapia concomitante comienza a ser el tratamiento estandar para múltiples patologías con lo que se incrementa la toxicidad de ambos tratamientos.

\section{Antagonistas de la 5-HT3}

Diversos estudios en pacientes sometidos a radioterapia apuntan a que el granisetrón y ondansetrón son significativamente mas efectivos que el placebo $u$ otros antieméticos. Lanciano ${ }^{11}$ compara la eficacia de $2 \mathrm{mg} /$ día de granisetrón frente a placebo como profiláctico en pacientes sometidos a radioterapia fraccionada sobre abdomen superior, demostrando su beneficio. Franzén ${ }^{6}$ presenta el beneficio de 8 
$\mathrm{mg} / 12$ horas de ondansetrón frente a placebo. Sin embargo, hay algunos puntos no señalados en ambos trabajos, tal como el sexo, la edad y en consumo de alcohol no vienes reflejados; no está claro si está distribuido uniformemente la posición de tratamiento en ambos brazos y si el tamaño de los campos de irradiación influye o no en los resultados ${ }^{12}$.

Estudios en fase II señalan que el dolasetrón es efectivo en la profilaxis de náuseas y vómitos radioinducidos. Bey ${ }^{13}$ compara varias dosis de dolasetrón frente a placebo obteniendo los mejores resultados en el grupo sometido a 0.3 $\mathrm{mg} / \mathrm{kg} /$ día iv. Fauser ${ }^{14}$ presenta un $65 \%$ de pacientes protegidos con $100 \mathrm{mg} /$ día vo sometidos a TBI y ciclofosfamida.

Existe poca literatura referente al uso de tropisetrón en nausea y vómitos radioinducidos ${ }^{15,16}$

Otras publicaciones han comparado granisetrón con metoclopramida. Prentice ${ }^{17}$ presenta un estudio randomizado a doble ciego entre $3 \mathrm{mg}$ de granisetrón y $20 \mathrm{mg}$ de metoclopramida en pacientes sometidos a TBI, obteniendo un 53\% sin emesis en el grupo de granisetrón y $13 \%$ en el grupo de metoclopramida.

Ondansetrón también ha demostrado ser mas efectivo que clorpromacina + dexametasona. Sykes ${ }^{18}$ presenta 66 pacientes sometidos a radioterapia abdominal, logrando un tasas del $70 \%$ sin náuseas en el grupo de ondansetrón frente al $28 \%$ sin nauseas en el otro grupo.

Spitzer ${ }^{19}$ concluye en su trabajo que $2 \mathrm{mg} /$ día vo de granisetrón y $8 \mathrm{mg} / 8$ horas de ondansetrón fueron efiaces en la prevención de las náuseas y vomitos radioinducidos en TBI hiperfraccionado sin claras diferencias entre ambos.

\section{Tratamiento antiemético en pacientes sometidos a irradiación}

\subsection{Interacción con otras drogas}

Se ha de tener en cuenta que en la inmensa mayoría de situaciones a la hora de instaurar un tratamiento antiemético nos encontramos con pacientes polimedicados y por ende se ha de valorar las posibles interacciones farmacológicas ${ }^{25}$, por ejemplo: el ondansetrón presenta interacción con los inhibidores de la recapatación de serotonina, utilizados estos como antidepresivos ${ }^{20,21}$, con algunos quimioterápicos como el cisplatino y la ciclofosfamida ${ }^{23,24}$, con determinados antibióticos como la rifampicina ${ }^{22}$. En cambio con el granisetrón no se ha descrito interacción con otros fármacos.

\subsection{Estreñimiento}

Diversos estudios han indicado que el uso continuado de antagonistas de la 5-HT3 pueden abocar en un estreñimiento ${ }^{2,8}$.

Lanciano ${ }^{11}$ presenta a 260 pacientes sometidos a irradiación abdominal. De los 126 con placebo, el 34\% presentó diarrea y el $0 \%$ estreñimiento, y de los 134 con granisetrón, el $27 \%$ presentó diarrea y el $19 \%$ estreñimiento.

\subsection{Problemas cardíacos}

Se ha sugerido que la radioterapia tiene el potencial de alterar la función cardiaca como efecto tardío 26 , también puede causar alteraciones electrocardiográficas como la prolongación del intervalo $\mathrm{QT}^{27}$, de ahí que el antiemético a usar es aconsejable que carezca de dichos efectos a fin de no agravar los problemas cardiacos de estos pacientes.

\subsection{Cansancio radioinducido}

El cansancio es uno de los síntomas mas frecuente e incómodo que pueden aparecer en el paciente neoplásico, y la radioterapia puede contribuir en ello. Smets ${ }^{28}$ presenta 250 pacientes sometidos a irradiación señala que el $43 \%$ presenta cansancio como uno de los tres síntomas mas incómodos. El cansancio puede verse potenciado por náuseas y vómitos. Por ello es importante el control de las nauseas y vómitos a fin de no aumentar la sensación de cansancio y por ende alterar la calidad de vida.

\section{Antieméticos en determinados grupos}

\subsection{Anciano}

La incidencia y mortalidad neoplásica es importante en los pacientes ancianos 9 , con una tasa del $60 \%$ en pacientes > 65 años $^{29}$, A pesar de ello no suelen incorporarse a los diversos estudios por su funcionalismo orgánico y la alta incidencia de polimedicación a fin de no alterar resultados. Por ello, los resultados de estos estudios no son claramente aplicados o aconsejados para los pacientes ancianos.

La incidencia de la comorbilidad que puede requerir la adición de un tratamiento farmacológico se incrementa con la edad ( $>3.6$ en pacientes $>65$ años) ${ }^{30}$. Gridelli $i^{31}$ presenta a 698 pacientes de edad >70 años tratados por carcinoma no microcítico de pulmón, el $60 \%$ presenta enfermedad cardiovascular, el $36 \%$ enfermedad respiratoria y el $32 \%$ enfermedad digestiva y/o hepática. El tratamiento en estas condiciones y otras como enfermedades locomotoras pueden causar efectos adversos indesesables ${ }^{32}$.

La enfermedad psiquiátrica es una importante consideración en los pacientes ancianos especialmente la depresión y los trastornos psicóticos ${ }^{33}$. Algunos antidepresivos interaccionan con antagonistas de la 5 -HT3. Koriech ${ }^{20}$ presenta a 3 pacientes oncológicos con fluoxitina se ve disminuida la eficacia antiemética del ondansetrón, por el contrario no ocurre con granisetrón ${ }^{34}$.

\subsection{Pacientes con alteraciones cardiacas, renales ó hepáticas}

Como ya hemos comentado es alta la incidencia de enfermedad cardiovascular entre los ancianos, al igual que alteraciones del funcionalismo hepatorrenal.

El granisetrón no precisa ajuste en caso de alteración hepatorrenal. Se han descrito alteraciones cardiovasculares y electrocardiográficas sin significado clínico ${ }^{35}$.

El ondansetrón no precisa ajuste en caso de insuficiencia renal, pero con grave alteración del funcionalismo hepático no debe sobrepasarse la dosis de $8 \mathrm{mg} /$ día. Se han descrito alteraciones electrocardiográficas (prolongación de QT), casos esporádicos de angina, hipotensión y taquicardias ${ }^{36-38}$.

La administración oral o parenteral de dolasetrón no se ve afectada en caso de insuficiencia hepática o renal sin precisar ajuste de la dosis. Puede causar alteraciones electrocardiográficas (alargamiento del intervalo QT) con importante repercusión clínica. Puede provocar arritmias ${ }^{39}$.

No se precisa de reducción de la dosis diaria de tropisetrón 


\section{G. Vázquez}

pero no se aconseja prolongar mas de 6 días el tratamiento en caso de alteración del funcionalismo hepatorrenal ${ }^{40}$.

\section{Criterios de consenso}

A) Radioterapia de alto riesgo emetógeno: TBI. Se aconseja el uso de un antagonista de la 5-HT3 con o sin esteroide antes de cada fracción y 24 horas después. Nivel de evidencia II y III. Grado de recomendación B y C.

La incidencia de emesis en este grupo es del 90-100\%. Las tasas de control emético con antagonistas de la $5 \mathrm{HT} 3$ en la $\mathrm{TBI}$ varían del 50 al $90 \% 17,41,42$. El papel de los esteroides asociados a los antagonistas de la 5 HT3 no ha sido estudiado. Existen trabajos ${ }^{43,44}$ que demuestran mayor efectividad de los antagonistas de la 5HT3 frente a metoclopramida ó fenotiacinas.

B) Radioterapia de moderado riesgo emetógeno: irradiación hemicorporal, irradiación del hemiabdomen superior irradiación abdominopélvica, irradiación en Mantle, irradiación craneoespinal, y radiocirugía cerebral.

Se aconseja el uso de un antagonista de la 5HT3 o antagonista dopaminérgico antes de cada fracción.

Nivel de evidencia II y III. Grado de recomendación B.

La incidencia de emesis en este grupo es del $30-90 \%$. Al parecer es mejor la profilaxis que el tratamiento en sí, y que el antagonista de la 5HT3 supera a la metoclopramida y fenotiazinas en este grupo de pacientes ${ }^{43,44}$ debido a las escasas diferencias entre los antagonistas de la 5HT3 y dopaminérgicos, algunos autores recomiendan estos últimos especialmente en pacientes sometidos a irradiación craneoespinal o irradiación hemicorporal inferior ${ }^{45}$. Otros autores ${ }^{46} \mathrm{su}^{-}$ gieren que la eficacia de los antagonistas de la 5 HT3 disminuye tras la primera semana de la irradiación. Los diversos trabajos señalan la eficacia de ambas drogas en este grupo de pacientes, con un mejor control con los antagonistas de la $5 \mathrm{HT} 3{ }^{44,45}$. En otros estudios ${ }^{47}$, el uso de canabinoides (nabilone y levonantradol) no ha demostrado adecuado control antiemético. Franzén ${ }^{45}$ presenta una eficacia similar entre dexametasona y antagonistas de la $5 \mathrm{HT} 3$ en pacientes sometidos a irradiación abdominal superior.

C) Radioterapia de bajo riesgo emetógeno: irradiación craneal, mama, cabeza y cuello, extremidades, tórax y pelvis.

Se aconseja el uso de antieméticos en caso de necesidad clínica, con antagonistas dopaminérgicos o de la 5 HT3. En caso de aparecer vómitos el uso profiláctico de estos fármacos es aconsejable.

Nivel de evidencia IV y V. Grado de recomendación B-D.

La incidencia de emesis es muy baja en este grupo de pacientes $(0-30 \%)$, por lo que parece razonable el uso de antieméticos en caso de presentarse. Con una escasez de artículos publicados en este grupo se aconseja el uso de antagonistas dopaminérgicos, reservando los antagonistas de la 5 HT3 en caso de fracaso de los anteriores ${ }^{3}$.

\section{Bibliografía}

1. Roila F, Ciccarese G, Paliadino MA, et al. Prevention of radiotherapy-induced emesis. Tumori 1998; 84:274-8.

2. Feyer PC, Stewart AL, Titbach OJ. Aetiology and prevention of emesis induced by radiotherapy. Support Cancer Care 1998; 6:253-60.
3. Gralla RJ, Osoba D, Kris MG, et al. Recommendations for the use of antiemetics: evidence-based, clinical practice guidelines. J Clin Oncol 1999; 17:2971-94.

4. Danjeoux CE; Rider WD; Fitzpatrick PJ. The acute radiation syndrome. A memorial to William Michael Court-Broun. Clin radiol 1979; 30:581-4.

5. Italian Group for antiemetic research in radiotherapy, radiation-induced emesis: a prospective observational multicenter Italian trial. Int J Rad Oncol Biol Phys 1999; 44:619-25.

6. Franzén L, Nyman H, Hagberg, et al. A randomized placebo controlled study with ondansetron in patients indergoing fraccionated radiotherapy. Ann Oncol 1996; 7:587-92.

7. Lawrence CC; Gilbert CJ, Peters WP. Evaluation of symptom distress in a bone marrow transplant outpatient environment. Ann Pharmacother 1996; 30:941-5.

8. Henriksson $R$, Lomberg $H$, Israelsson $G$, et al. The effect of ondansetron on radiation-induced emesis and diarrhoea. Acta Oncol 1992; 31:767-9.

9. Yancik R, Ries LAG. Aging and cancer in America. Hematol Oncol Clin North Am 2000; 14:17-23.

10. MASCC, Prevention of chemotherapy- and radiotherapy-induced emesis: results of the Perugia Consensus Conference. Ann Oncol 1998; 9:811-9.

11. Lanciano R, Sherman DM, Michalski J, et al. The efficacy and safety of once-daily Kytril( (granisetrón hydrochloride) tablets in the prophylaxis of nausea and emesis following fractionated upper abdominal radiotherapy. Cancer Invest 2001;19:763-72.

12. Aapro M. Radiotherapy and drugs: "setrons" once again. Editorial. Ann Oncol 1996; 7:553-4.

13. Bey $P$, Wilkinson PM, Resbeut $M$, et al. A double-blind, placebo-controlled trial of i.v. dolasetron mesilate in the prevention of radiotherapy-induced náusea and vomiting in cancer patients. Support Care Cancer 1996; 4:378-83.

14. Fauser AA, Russ W, Bischoff M. Oral ondansetron mesilate (MDL 73,147EF) for the control of emesis during fractionated total-body irradiation and high-dose cyclophosphamide in $\mathrm{pa}^{-}$ tients undergoing allogeneic bone marrow transplantation. Support Care Cancer 1997; 5:219-22.

15. Sorbe B, Berglind AM. Tropisetron, a new 5-HT3-receptor antagonist, in the prevention of radiation-induced nausea, vomiting and diarrhoea. Drugs 1992; 43(s3):33-39

16. Miralbell R, Coucke P, Behrouz, et al. nausea and vomiting in fractionated radiotherapy: a prospective on-demand trial of tropisetron rescue for non-responders to metoclopramide. Eur J cancer 1995; 31A:1461-4.

17. Prentice HG, Cunningham S, Gandhi L, et al. Granisetron in the prevention of irradiation-induced emesis. Bone Marrow Transplant 195; 15:445-8.

18. Sykes AJ, Kiltie AE, Stewart AL. Ondansetron versus a chlorpromazine and dexamethasone combination for the prevention of nausea and vomiting: a prospective, randomized study to assess efficacy, cost effectiveness and quality of life following single-fraction radiotherapy. Support care Cancer 1997; 5:500-3.

19. Spitzer TR, Friedman CJ, Bushnell W, et al. Double-blind, randomized, parallel-group study on the efficacy and safety of oral granisetrón and oral ondansetron in the prophylaxis of nausea and vomiting in patients receiving hyperfractionated total body irradiation. Bone marrow Transplant 2000; 26:203-10.

20. Koriech OM. Fluoxetine treatment compromises the antiemetic efficacy of ondansetron in cancer patients. Clin Oncol 1995; 7:371-2. 
21. Stanford BJ; Stanford SC. Postoperative delirium indicating an adverse drug interaction involving the selective serotonin reuptake inhibitor, paroxetine. J Psychopharmacol 1999; 13:3137.

22. Hayes E. Cyłochrome P450. http:/www.anaesthetist.com/physiol/basics/metabol/cyp/o.htm

23. Cagnoni PJ, Matthes S, Day TC, et al. Modification of the pharmacokinetics of high-dose cyclophosphamide end cisplatin by antiemetics. Bone Marrow Transplant 1999; 24:1-4.

24. Gilbert CJ, Petros WP, Vredenburgh J, et al. Pharmacokinetic interaction between ondansetron and cyclophosphamide during high-dose chemotherapy for breast cancer. Cancer Chemother Pharmacol 1998; 42:497-503.

25. Karas Jr S. The potential for drug interactions. Ann Emerg med $1981 ; 10: 627-30$.

26. Adams MJ, Hardenbergh PH, Constine LS, et al. radiation-associated cardiovascular disease. Crit Rev Oncol Hematol 2003; 45:55-75.

27. Biller H, Koop EA, Prignitz R. ECG-changes following postoperative irradiation of patients with mammary carcinoma. Strahlentherapie 1979; 155:541-8.

28. Smets $E M$, Visser MR, Willems-Groot AF, et al. fatigue and radiotherapy: $\mathrm{A}$ experience in patients undergoing treatment. $\mathrm{Br} J$ Cancer 1998; 78:899-906

29. Yancik R, Ganz PA, Varricchio CG, et al. Perspectives on comorbidity and cancer in older patients: approaches to expand the knowledge base. J Clin Oncol 2001; 19;1147-51.

30. Yancik R. Cancer burden in the aged. An epidemiological and demographic overview. Cancer 1997; 80:1273-83.

31. Gridelli C, Perrone F, Gallo C, et al. Chemotherapy for elderly patients with advanced non-small-cell lung cancer: the Multicenter Italian lung cancer in the Ederly Study (MILES) phase III randomized trial. J Natl Cancer Inst 2003; 95:362-72.

32. Ashkanani $F$, Heys SD, Eremin O. The management of cancer in the elderly. J R Coll Surg Edinb 1999; 44:2-10.

33. Angelino AF, Treisman GJ. Major depresión and demoralization in cancer patients: diagnostic and treatment considerations. Support Care Cancer 2001; 9:344-9.

34. Newberry NR; Watkins C; Sprosen T, et al. BRL 46470 potently antagonizes neural responses activated by 5 - $\mathrm{HT} 3$ receptors.
35. Kytri ${ }^{\circledR}$ (granisetrón hydrochloride), Prescribing Information, Roche Laboratories Inc, Nutley, New Jersey, USA 2000.

36. Zofra ${ }^{\circledR}$ (ondansetron hydrochloride), Prescribing Information, Reseach Triangle Park, NC, USA 2001.

37. Lifsey DS, Gralla RJ, Clark RA, et al. Electrocardiographic changes with serotonin antagonist antiemetics: rate of occurrence and clinical relevance. Proc Am Soc Clin Oncol 1993; 12: 463.

38. Boike SC, Ilson B, Zariffa N, et al. cardiovascular effects of i.v. granisetrón at two administration rates and of ondansetron in healthy adults. Am J Helath Syst Pharm 1997; 54:1172-6.

39. Anzemet ${ }^{\circledR}$ (dolasetron mesylate), Prescribing Information, Aventis Pharmaceuticals Inc, Kansas City, MO, USA 2000.

40. Navoban ${ }^{\circledR}$, Product Information Ltd, North Ryde NSW, Australia 1997.

41. Spitzer TR, Grunberg SM, Dicato MA. Antiemetic strategies for high-dose chemotherapy-induced nausea and vomiting. Support Care Cancer 1998;6:233-36.

42. Kirkbride P, Pater J, Zee B, et al. A phase III study of the efficacy of dexamethasone (DEX) in the prophylaxis of radiation induced emesis (RIE). Proc Am Soc Clin Oncol 1998; 17:51A.

43. Tiley C, Powles R, Catalano J, et al. Results of a double-blind placebo controlled study of ondansetron as an antiemetic during total body irradiation in patients undergoing bone marrow transplantation. Leuk Lymphoma 1992; 7:317-21.

44. Priestman TJ, Roberts JT, Upadhyaya BK. A prospective randomized double-blind trial comparing ondansetron versus prochlor-perazine for the prevention of nausea and vomiting in patients undergoing fractionated radiotherapy. Clin Oncol 1993; 5:358-63.

45. Franzen L, Nyman J, Hagberg $\mathrm{H}$, et al. A randomized placebo controlled study with ondansetron in patients undergoing fractionated radiotherapy. Ann Oncol 1996;7: 587-92.

46. Priestman SG, Priestman TJ, Canney PA. A double-blind randomized cross-over comparison of nabilona and metoclopramide in the control of radiation-induced nausea. Clin radiol 1987; 38:543-44

47. Ungerleider JT, Andrysiak TA, Fairbanks LA, et al. Terahydrocannabinol vs. prochlorperazine: the effects of two antiemetics on patients undergoing radiotherapy. Radiology 1984; 150: 598-9. 